\title{
The role of effective triplet interactions in charged colloidal suspensions
}

\author{
H Löwen $\dagger$ and E Allahyarov $\ddagger$ \\ Institut für Theoretische Physik II, Heinrich-Heine-Universität Düsseldorf, Universitätsstraße 1, \\ D-40225 Düsseldorf, Germany
}

Received 8 December 1997, in final form 13 March 1998

\begin{abstract}
The traditional theory of the effective interactions between charged colloidal particles ('macro-ions') treats the screening of the counter-ions on a linear response level resulting in the well-known screened Coulomb pair potential proposed by Derjaguin, Landau, Verwey and Overbeek. In general, however, due to non-linear counter-ion screening, the effective interactions involve many-body contributions. Using molecular dynamics simulation of the highly asymmetric primitive model with three macro-ions, we calculate the effective triplet forces and compare them with the pairwise contributions. Near touching of a macro-ion triplet, the triplet interaction represents a significant attractive correction to the repulsive pairwise interaction while it is small for large inter-particle separations. Furthermore, we compare our simulation results for the triplet forces with an analytical expression gained from a density functional perturbation approach. Upon performing a suitable renormalization, we find reasonable agreement for situations of weak and strong coupling as well as for situations with added salt ions.
\end{abstract}

\section{Introduction}

In the theory of simple liquids [1-3] one typically works with radially symmetric pair potentials $V(R), R$ denoting the distance between the centres of two particles. The most prominent examples for such pairwise interactions are the hard-sphere fluid, which is completely described by excluded-volume interactions, and the classical plasma, for which $V(R) \sim 1 / R$. Furthermore, the interaction between dilute rare gases is well described by the Lennard-Jones pair potential while the interaction between chargestabilized colloids is frequently taken to be the pair potential proposed by Derjaguin, Landau, Verwey and Overbeek (DLVO) more than fifty years ago [4, 5]. In the latter two cases, however, one should bear in mind that the system consists of more than a single component. There are additional degrees of freedom, namely those of the polarizable electrons in the case of rare gases and those of the counter-ions in the case of charged colloids. The pair potential has to be understood as an effective interaction obtained by integrating out the additional degrees of freedom using a thermal canonical average [6]. Since this average is highly non-linear, in general, the effective interactions

$\dagger$ Also at: Institut für Festkörperforschung, Forschungszentrum Jülich, D-52425 Jülich, Germany.

$\ddagger$ Permanent address: Institute for High Temperatures, Russian Academy of Sciences, Izhorskaya Street 13/19, 127412 Moscow, Russia. 
involve many-body terms, i.e. there are pair, triplet, quadruplet contributions etc; see references $[7,8]$.

It is only for low densities that the pairwise terms dominate the total interactions. For finite density, the triplet and higher-order forces can contribute significantly. In the case of rare gases, for instance, at intermediate densities, high-accuracy measurements of the fluid structure factor can only be understood completely by adding the so-called Axilrod-Teller triplet interaction [9] to the usual pairwise interaction; see e.g. [10]. It is clear that an explicit representation of the triplet potential is much more complicated than the pairwise part $V(R)$, which only depends on one scalar variable, $R$. In fact, the triplet interactions depend on three scalar variables, namely the two distances between two particles and the angle between their directions.

While the triplet interactions are well studied and understood for rare-gas fluids and liquids, much less is known about the role of triplet interactions in charged colloidal suspensions. Usually one focuses on the pairwise level and tries to incorporate manybody effects into effective parameters of the pairwise interactions which then depend on the thermodynamic state of the system [11]. More insight into the many-body interactions can be gained from Car-Parrinello-type 'ab initio' calculations [8, 12]. In fact, one can obtain an optimal effective pair potential from these data which describes the forces well [13]. The aim and motivation of the present paper is different: we want to extract and study the triplet interactions alone, aiming to separate them from quadruplet and higher-order interactions. Therefore we study a situation where only three macro-ions are involved. They are placed in a cubic box surrounded by their counter-ions and eventually by added salt ions. Using molecular dynamics simulations techniques and the primitive model of highly asymmetric electrolytes, we are able to calculate the total effective forces acting on the macro-ions. By a reference calculation with two macro-ions, we obtain the pairwise part. By subtracting the results, we are able to access the triplet forces directly, i.e. to separate them from the pairwise contributions. As a result, the triplet forces only become relevant for a situation where three macro-ions are close together, i.e. for a nearly touching triplet. We show that the triplet forces are attractive and can become comparable to the repulsive pairwise forces for a nearly touching triplet. Furthermore, we test an analytical expression for the triplet forces based on the density functional perturbation approach around the DLVO solution which was originally proposed for the salt-free case in reference [8]. We show that, if a suitable renormalization is also performed, this theory works well in all of the situations considered even for a situation with added salt ions. The computational technique that we employed is similar to that used in previous work [14-16].

We remark that triplet interactions are different from triplet correlations. Of course, even for pairwise interactions there are non-trivial triplet correlations [17]. Linse [18] has used computer simulation data obtained for the primitive model with moderate charge asymmetries in order to calculate the triplet correlations for the macro-ions. Although the numerical technique and the model that he used are identical to ours, the aim of the paper and the results are different. Linse used the triplet correlations functions as diagnostics to detect the effects stemming from triplet forces, while we calculate these forces directly. Furthermore, our approach is feasible also for higher charge asymmetries typical for real samples of charged colloidal suspensions.

Our paper is organized as follows. In section 2 we define the primitive model and give the definition of the effective force gained from statistical mechanics. We then briefly describe our simulation technique in section 3 . The density functional perturbation theory is derived in section 4 . In section 5 we present our results. Section 6 , finally, is devoted to a discussion and a forward look. 


\section{The primitive model and the definition of the forces}

We consider $N_{m}$ macro-ions of charge $-Z e(Z>0)$ and radius $R_{m}$ in a volume $\Omega$ at temperature $T$; hence $\rho_{m}=N_{m} / \Omega$ is the macro-ion number density. Here $e$ is the (positive) elementary charge. Together with the macro-ions, we have $N_{c}$ counter-ions of opposite charge $+q e(q>0)$. Global charge neutrality fixes $N_{c}$ as follows:

$$
N_{c}=\left|\frac{Z}{q}\right| N_{m}
$$

which implies for the counter-ion number density

$$
\rho_{c}=\frac{Z}{q} \rho_{m} .
$$

We also consider added 1:1 salt ions providing co-ions of charge $-q e$ and additional counterions. The co-ion number $N_{s}$ is an additional free parameter of the model which vanishes in the salt-free case. The total counter-ion and co-ion densities $\rho_{+}$and $\rho_{-}$are given by

$$
\rho_{+}=\rho_{-}+\rho_{c} \quad \rho_{-}=\frac{N_{s}}{\Omega}
$$

where we assume that salt counter-ions and counter-ions stemming from the macro-ion are indistinguishable.

Within the primitive model, the interactions between the macro-ions, counter-ions and salt ions are

$$
\begin{aligned}
& V_{m m}(R)= \begin{cases}\frac{Z^{2} e^{2}}{\varepsilon R} & \text { for } R \geqslant 2 R_{m} \\
\infty & \text { for } R<2 R_{m}\end{cases} \\
& V_{m i}(R)= \begin{cases}-\frac{Z q_{i} e^{2}}{\varepsilon R} & \text { for } R \geqslant R_{m}+\sigma_{i} / 2 \\
\infty & \text { for } R<R_{m}+\sigma_{i} / 2\end{cases} \\
& V_{i j}(R)= \begin{cases}\frac{q_{i} q_{j} e^{2}}{\varepsilon R} & \text { for } R \geqslant\left(\sigma_{i}+\sigma_{j}\right) / 2 \\
\infty & \text { for } R<\left(\sigma_{i}+\sigma_{j}\right) / 2\end{cases}
\end{aligned}
$$

Here $R$ is the separation distance, $\varepsilon$ is the dielectric constant of the solvent, and the labels $i$ and $j$ can assume the values + and - , with $q_{+} \equiv q$ and $q_{-} \equiv-q . \sigma_{+} \equiv \sigma_{-}$are the microscopic counter- and co-ion diameters.

The total effective force $\boldsymbol{F}_{l}$ acting on the macro-ion at position $\boldsymbol{R}_{l}\left(l=1, \ldots, N_{m}\right)$ is the canonical average with respect to the microscopic ions $[8,14]$ :

$$
\boldsymbol{F}_{l}\left(\left\{\boldsymbol{R}_{k}\right\}\right)=\boldsymbol{F}_{l}^{\text {dir }}\left(\left\{\boldsymbol{R}_{k}\right\}\right)+\boldsymbol{F}_{l}^{\text {ind }}\left(\left\{\boldsymbol{R}_{k}\right\}\right) .
$$

The direct force is given as

$\boldsymbol{F}_{l}^{d i r}=-\nabla_{\boldsymbol{R}_{l}} \sum_{\substack{k=1 \\ k \neq l}}^{N_{m}} V_{m m}\left(\left|\boldsymbol{R}_{l}-\boldsymbol{R}_{k}\right|\right)$

and the indirect part is

$$
\begin{aligned}
& \boldsymbol{F}_{l}^{\text {ind }}=\int_{\Omega} \mathrm{d}^{3} \boldsymbol{r} {\left[\rho_{+}^{(0)}\left(\boldsymbol{r},\left\{\boldsymbol{R}_{k}\right\}\right)\left(-\nabla_{\boldsymbol{R}_{l}}\right) V_{m+}\left(\left|\boldsymbol{r}-\boldsymbol{R}_{l}\right|\right)\right.} \\
&\left.+\rho_{-}^{(0)}\left(\boldsymbol{r},\left\{\boldsymbol{R}_{k}\right\}\right)\left(-\nabla_{\boldsymbol{R}_{l}}\right) V_{m-}\left(\left|\boldsymbol{r}-\boldsymbol{R}_{l}\right|\right)\right] .
\end{aligned}
$$


Here, $\rho_{ \pm}^{(0)}\left(\boldsymbol{r},\left\{\boldsymbol{R}_{k}\right\}\right)$ are the equilibrium counter- and co-ion density profiles in the presence of the macro-ions. These key quantities can be obtained following two different routes, either from density functional theory or from computer simulation. Their statistical mechanical definition is

$$
\begin{aligned}
& \rho_{+}^{(0)}\left(\boldsymbol{r},\left\{\boldsymbol{R}_{k}\right\}\right)=\left\langle\sum_{j=1}^{N_{s}+N_{c}} \delta\left(\boldsymbol{r}-\boldsymbol{r}_{j}^{+}\right)\right\rangle \\
& \rho_{-}^{(0)}\left(\boldsymbol{r},\left\{\boldsymbol{R}_{k}\right\}\right)=\left\langle\sum_{j=1}^{N_{s}} \delta\left(\boldsymbol{r}-\boldsymbol{r}_{j}^{-}\right)\right\rangle .
\end{aligned}
$$

Here the canonical average over the positions of the microscopic ions is defined as

$$
\begin{aligned}
\langle\cdots\rangle=\frac{1}{Z}\left\{\prod_{i=1}^{N_{s}+N_{c}} \int_{\Omega} \mathrm{d}^{3} r_{i}^{+}\right\}\left\{\prod_{j=1}^{N_{s}} \int_{\Omega} \mathrm{d}^{3} r_{j}^{-}\right\}(\cdots) \\
\quad \times \exp \left(-\left[U_{m+}+U_{m-}+U_{++}+U_{+-}+U_{--}\right] / k_{B} T\right)
\end{aligned}
$$

where

$$
U_{m i}=\sum_{v=1}^{N_{i}} \sum_{k=1}^{N_{m}} V_{m i}\left(\left|\boldsymbol{r}_{v}^{i}-\boldsymbol{R}_{k}\right|\right)
$$

and

$$
U_{i j}=\left(1-\frac{1}{2} \delta_{i j}\right) \sum_{\nu=1}^{N_{i}} \sum_{\mu=1}^{N_{j}} V_{i j}\left(\left|\boldsymbol{r}_{v}^{i}-\boldsymbol{r}_{\mu}^{j}\right|\right)
$$

with $i, j=+,-$ and $N_{+}=N_{c}+N_{s}, N_{-} \equiv N_{s}$. Finally, in (11), $Z$ guarantees correct normalization, $\langle 1\rangle=1$.

\section{Molecular dynamics simulation of the triplet forces}

Here we consider $N_{m}=3$ particles in an cubic box with impenetrable walls. The box length $L$ is fixed by the macro-ion density $\rho_{m}$, such that $L=\sqrt[3]{3 / \rho_{m}}$. The position of the macro-ion triplet is such that it forms an equilateral triangle of length $R$; see figure 1 . Explicitly, the positions $\boldsymbol{R}_{1}, \boldsymbol{R}_{2}, \boldsymbol{R}_{3}$ of the macro-ions are

$$
\begin{aligned}
& \boldsymbol{R}_{1}=\left(\frac{L}{2}, \frac{L}{2}-\frac{R}{\sqrt{6}}, \frac{L}{2}-\frac{R}{\sqrt{6}}\right) \\
& \boldsymbol{R}_{2}=\left(\frac{L}{2}-\frac{R}{2}, \frac{L}{2}+\frac{R}{2 \sqrt{6}}, \frac{L}{2}+\frac{R}{2 \sqrt{6}}\right) \\
& \boldsymbol{R}_{3}=\left(\frac{L}{2}+\frac{R}{2}, \frac{L}{2}+\frac{R}{2 \sqrt{6}}, \frac{L}{2}+\frac{R}{2 \sqrt{6}}\right) .
\end{aligned}
$$

We have only considered such a triangle-shaped triplet configuration since the triplet forces are expected to be most pronounced here. Of course, a linear arrangement of three particles is also conceivable. In such a linear configuration, however, triplet interactions are expected to vanish, since the two outermost particles practically do not interact with each other.

If the particles are sufficiently far away from the walls, the direction of the total effective force to particle 1 coincides with the difference vector $\boldsymbol{d}=\boldsymbol{R}_{1}-\frac{1}{3}\left(\boldsymbol{R}_{1}+\boldsymbol{R}_{2}+\boldsymbol{R}_{3}\right)$ of the centre of mass of the triplet and the position of particle 1; see again figure 1. This follows immediately from symmetry. Also, the magnitudes of the forces $\boldsymbol{F}_{1}, \boldsymbol{F}_{2}, \boldsymbol{F}_{3}$ are the same, 


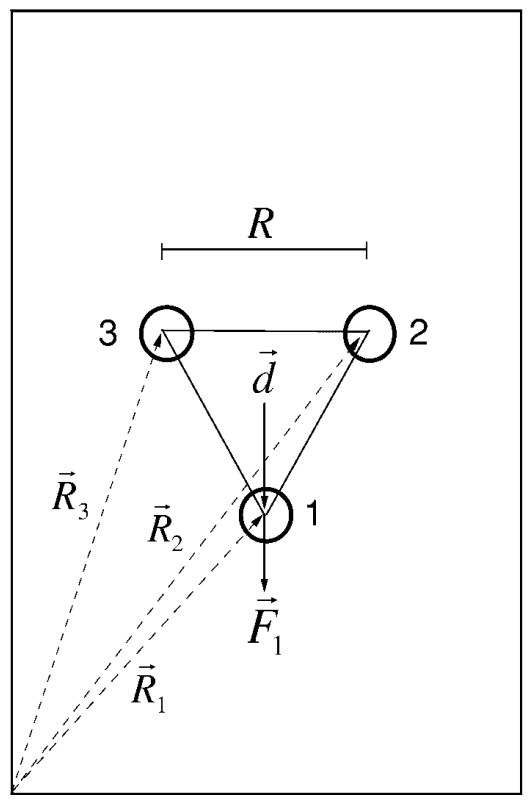

Figure 1. The triangular geometry, formed by three macro-ions labelled 1, 2, 3. Their positions $\boldsymbol{R}_{1}, \boldsymbol{R}_{2}, \boldsymbol{R}_{3}$ are also sketched, and the direction of the total effective force $\boldsymbol{F}_{1}(R)$, acting on the first macro-ion, is shown as well as the difference vector $\boldsymbol{d}$.

$\left|\boldsymbol{F}_{1}\right|=\left|\boldsymbol{F}_{2}\right|=\left|\boldsymbol{F}_{3}\right|$. Hence it is sufficient to consider $\boldsymbol{F}_{1}$ only. We define the projected force via

$$
F(R) \equiv \boldsymbol{F}_{1}(R) \cdot \boldsymbol{d} /|\boldsymbol{d}| .
$$

Positive $F(R)$ means repulsion, negative $F(R)$ attraction.

Using standard molecular dynamics simulations [2] we have computed the statistical averages and the distance-resolved forces on the particles. In order to separate effects from pair and triplet contributions we have performed the calculations for $N_{m}=2$ and $N_{m}=3$. The calculations for $N_{m}=2$ are similar to those in $[14,15]$. Results are given and discussed in section 5 .

\section{Density functional perturbation theory}

Let us now follow the route of density functional theory to predict the triplet forces. The equilibrium densities $\rho_{ \pm}^{(0)}\left(\boldsymbol{r},\left\{\boldsymbol{R}_{k}\right\}\right)$ can be obtained from minimizing the free-energy density functional $\mathcal{F}\left(\left[\rho_{+}(\boldsymbol{r})\right],\left[\rho_{-}(\boldsymbol{r})\right],\left\{\boldsymbol{R}_{k}\right\}\right)$ with the constraint of given total ion numbers

$$
N_{+}=\int_{\Omega} \mathrm{d}^{3} r \rho_{+}(\boldsymbol{r}) \quad N_{-}=\int_{\Omega} \mathrm{d}^{3} r \rho_{-}(\boldsymbol{r}) .
$$

Within the local density approximation (at the Poisson-Boltzmann level), this functional can be written as $[3,8]$

$$
\begin{gathered}
\mathcal{F}\left(\left[\rho_{+}(\boldsymbol{r})\right],\left[\rho_{-}(\boldsymbol{r})\right],\left\{\boldsymbol{R}_{k}\right\}\right)=\mathcal{F}_{i d}^{+}\left[\rho_{+}(\boldsymbol{r})\right]+\mathcal{F}_{i d}^{-}\left[\rho_{-}(\boldsymbol{r})\right]+\mathcal{F}_{\text {ext }}\left(\left[\rho_{+}(\boldsymbol{r})\right],\left[\rho_{-}(\boldsymbol{r})\right],\left\{\boldsymbol{R}_{k}\right\}\right) \\
+\frac{1}{2} \int_{\Omega} \mathrm{d}^{3} r \int_{\Omega} \mathrm{d}^{3} r^{\prime}\left(\rho_{+}(\boldsymbol{r})-\rho_{-}(\boldsymbol{r})\right)\left(\rho_{+}\left(\boldsymbol{r}^{\prime}\right)-\rho_{-}\left(\boldsymbol{r}^{\prime}\right)\right) V_{++}\left(\left|\boldsymbol{r}-\boldsymbol{r}^{\prime}\right|\right)
\end{gathered}
$$


where

$$
\mathcal{F}_{i d}^{ \pm}\left[\rho_{ \pm}(\boldsymbol{r})\right]=k_{B} T \int_{\Omega} \mathrm{d}^{3} r \rho_{ \pm}(\boldsymbol{r})\left(\ln \left[\Lambda_{ \pm}^{3} \rho_{ \pm}(\boldsymbol{r})\right]-1\right) .
$$

Here $\Lambda_{ \pm}$are the thermal de Broglie wavelengths of the microscopic ions. Furthermore, the coupling between the microscopic and mesoscopic ions is given by

$\mathcal{F}_{\text {ext }}\left(\left[\rho_{+}(\boldsymbol{r})\right],\left[\rho_{-}(\boldsymbol{r})\right],\left\{\boldsymbol{R}_{k}\right\}\right)=\int_{\Omega} \mathrm{d}^{3} r \sum_{j=1}^{N_{m}}\left(\rho_{+}(\boldsymbol{r}) V_{m+}\left(\left|\boldsymbol{r}-\boldsymbol{R}_{j}\right|\right)+\rho_{-}(\boldsymbol{r}) V_{m-}\left(\left|\boldsymbol{r}-\boldsymbol{R}_{j}\right|\right)\right)$.

Since $\mathcal{F}_{i d}^{ \pm}$is non-quadratic in the densities, the minimizing equations

$$
\frac{\delta \mathcal{F}}{\delta \rho_{ \pm}}=\mu^{ \pm}
$$

are non-linear and cannot be solved analytically, $\mu^{ \pm}$being the corresponding Lagrange multiplier used to ensure the prescribed overall density.

An analytical solution equivalent to the DLVO theory can be obtained for point particles $\left(R_{m}=\sigma_{+}=\sigma_{-}=0\right)$ and a truncated quadratic expansion of $\mathcal{F}_{i d}^{ \pm}$. We may functionally expand $\mathcal{F}_{i d}^{ \pm}$around the mean densities $\rho_{ \pm}$as follows:

$$
\begin{aligned}
\mathcal{F}_{i d}^{ \pm}\left[\rho_{ \pm}(\boldsymbol{r})\right]= & \mathcal{F}_{0}+k_{B} T \int_{\Omega} \mathrm{d}^{3} r\left(\ln \left(\Lambda_{ \pm}^{3} \rho_{ \pm}\right)\left(\rho_{ \pm}(\boldsymbol{r})-\rho_{ \pm}\right)\right. \\
& \left.+\sum_{n=2}^{\infty} \frac{(-1)^{n}}{n(n-1)} \frac{1}{\rho_{ \pm}^{n-1}}\left(\rho_{ \pm}(\boldsymbol{r})-\rho_{ \pm}\right)^{n}\right) .
\end{aligned}
$$

By admitting only quadratic terms ( $n=2$ in the sum of (20)) we can solve the minimization equation (19) by Fourier transformation, getting a linear superposition of Yukawa orbitals for the equilibrium densities

$$
\rho_{+}^{(0)}\left(\boldsymbol{r},\left\{\boldsymbol{R}_{k}\right\}\right)=\frac{2 \rho_{+} \rho_{-}}{\rho_{+}+\rho_{-}}+\frac{Z}{q} \frac{\kappa_{+}^{2}}{4 \pi} \sum_{j=1}^{N_{m}} \frac{\exp \left(-\kappa\left|\boldsymbol{r}-\boldsymbol{R}_{j}\right|\right)}{\left|\boldsymbol{r}-\boldsymbol{R}_{j}\right|}
$$

and

$$
\rho_{-}^{(0)}\left(\boldsymbol{r},\left\{\boldsymbol{R}_{k}\right\}\right)=\frac{2 \rho_{+} \rho_{-}}{\rho_{+}+\rho_{-}}-\frac{Z}{q} \frac{\kappa_{-}^{2}}{4 \pi} \sum_{j=1}^{N_{m}} \frac{\exp \left(-\kappa\left|\boldsymbol{r}-\boldsymbol{R}_{j}\right|\right)}{\left|\boldsymbol{r}-\boldsymbol{R}_{j}\right|}
$$

where

$$
\kappa^{2}=\frac{4 \pi e^{2} q^{2}\left(\rho_{+}+\rho_{-}\right)}{\varepsilon k_{B} T} \quad \kappa_{ \pm}^{2}=\frac{4 \pi e^{2} q^{2} \rho_{ \pm}}{\varepsilon k_{B} T} .
$$

The total effective forces are then obtained by using their definitions (6)-(8) from section 2. As a result, in this approximation,

$$
\boldsymbol{F}_{l} \cong \boldsymbol{F}_{l}^{(\mathrm{DLVO})}:=-\nabla_{\boldsymbol{R}_{l}}\left(\sum_{\substack{k=1 \\ k \neq l}}^{N_{m}} \frac{Z^{2} e^{2}}{\varepsilon} \frac{\exp \left(-\kappa\left|\boldsymbol{R}_{k}-\boldsymbol{R}_{l}\right|\right)}{\left|\boldsymbol{R}_{k}-\boldsymbol{R}_{l}\right|}\right)
$$

which coincides with the DLVO theory prediction for $R_{m}=0$. Obviously a quadratic expansion in $\mathcal{F}_{i d}$ (or equivalently linear screening theory) leads necessarily to pairwise effective forces. As it stands, the quadratic expansion in $\mathcal{F}_{i d}^{ \pm}$only works for weak density modulations, $\left|\rho_{ \pm}(\boldsymbol{r})-\rho_{ \pm}\right| / \rho_{ \pm} \ll 1$. 
The key idea of the density functional perturbation approach is to realize that the effective indirect forces stem from the potential

$$
\mathcal{F}\left(\left[\rho_{+}^{(0)}\left(\boldsymbol{r},\left\{\boldsymbol{R}_{k}\right\}\right)\right],\left[\rho_{-}^{(0)}\left(\boldsymbol{r},\left\{\boldsymbol{R}_{k}\right\}\right)\right],\left\{\boldsymbol{R}_{k}\right\}\right) .
$$

That is,

$$
\boldsymbol{F}_{l}^{i n d}=-\nabla_{\boldsymbol{R}_{1}} \mathcal{F}\left(\left[\rho_{+}^{(0)}\left(\boldsymbol{r},\left\{\boldsymbol{R}_{k}\right\}\right)\right],\left[\rho_{-}^{(0)}\left(\boldsymbol{r},\left\{\boldsymbol{R}_{k}\right\}\right)\right],\left\{\boldsymbol{R}_{k}\right\}\right)
$$

We now assume in the spirit of perturbation theory that the leading correction to the linear screening theory is given by the $n=3$ term in the sum of (20) evaluated at the unperturbed densities $\rho_{ \pm}^{(0)}\left(\boldsymbol{r},\left\{\boldsymbol{R}_{k}\right\}\right)$ obtained from the linearized theory. Formally this is justified for weak density modulations, i.e. for $\left|\rho_{ \pm}(\boldsymbol{r})-\rho_{ \pm}\right| / \rho_{ \pm} \ll 1$. We remark that we neglect the contributions from higher orders $(n>3)$ which also contribute to the pair and triplet forces. Therefore this leaves open the question of whether a possible inadequacy of the density functional approach is due to the Poisson-Boltzmann approximation or the truncation. This gives rise to the following correction to the DLVO forces (24):

$$
\boldsymbol{F}_{l} \cong \boldsymbol{F}_{l}^{(\mathrm{DLVO})}+\boldsymbol{F}_{l}^{(\text {pert })}
$$

where

$$
\boldsymbol{F}_{l}^{(\text {pert })} \equiv+\nabla_{\boldsymbol{R}_{l}} \frac{k_{B} T}{6} \int_{\Omega} \mathrm{d}^{3} r\left\{\frac{1}{\rho_{+}^{2}}\left[\rho_{+}^{(0)}\left(\boldsymbol{r},\left\{\boldsymbol{R}_{k}\right\}\right)-\rho_{+}\right]^{3}+\frac{1}{\rho_{-}^{2}}\left[\rho_{-}^{(0)}\left(\boldsymbol{r},\left\{\boldsymbol{R}_{k}\right\}\right)-\rho_{-}\right]^{3}\right\} .
$$

Here $\rho_{ \pm}^{(0)}$ is analytically given by (21), (22). Equation (27) constitutes our main result. Since only cubes of linearly superimposed Yukawa orbitals occur in (27), we are indeed dealing with additional pair and triplet forces.

A final remark is in order for $R_{m}>0$. For this case we assume that the ion density is zero inside the macro-ion core. Furthermore, we include the DLVO size correction. Hence our final result for the triplet forces is the expression (27) together with the density fields

$$
\begin{aligned}
& \rho_{+}^{(0)}\left(\boldsymbol{r},\left\{\boldsymbol{R}_{k}\right\}\right)=\frac{2 \rho_{+} \rho_{-}}{\rho_{+}+\rho_{-}}+\frac{\kappa_{+}^{2}}{\kappa^{2}} \sum_{j=1}^{N_{m}} \varphi\left(\left|\boldsymbol{r}-\boldsymbol{R}_{j}\right|\right) \\
& \rho_{-}^{(0)}\left(\boldsymbol{r},\left\{\boldsymbol{R}_{k}\right\}\right)=\frac{2 \rho_{+} \rho_{-}}{\rho_{+}+\rho_{-}}-\frac{\kappa_{-}^{2}}{\kappa^{2}} \sum_{j=1}^{N_{m}} \varphi\left(\left|\boldsymbol{r}-\boldsymbol{R}_{j}\right|\right)
\end{aligned}
$$

and the orbital function

$$
\varphi(r)= \begin{cases}\frac{Z}{q} \frac{\kappa^{2}}{4 \pi} \frac{\exp \left(\kappa R_{m}\right)}{1+\kappa R_{m}} \frac{\exp (-\kappa r)}{r} & \text { for } r>R_{m} \\ 0 & \text { otherwise. }\end{cases}
$$

We finally note that the theory presented had already been proposed in reference [8] but only for the salt-free case and vanishing macro-ion radius. In fact, our result reduces to that given in reference [8] on setting $\rho_{-} \equiv 0$ and $R_{m} \equiv 0$.

The density functional perturbation approach yields for the special case considered in section 3 the following expression for the force $F \equiv \boldsymbol{F}_{1} \cdot \boldsymbol{d} / \boldsymbol{d}$ :

$$
F \cong F^{(\mathrm{DLVO})}+F^{(\text {pert })}
$$

where the perturbed part $F^{(\text {pert })}$ has a pairwise and a triplet part:

$$
F^{(\text {pert })}=F_{\text {pert }}^{(2)}+F_{\text {pert }}^{(3)} .
$$


Table 1. Theoretical and simulation data for the triplet forces together with the statistical error of the simulation for runs A, B and C. The other parameters are presented in section 5. Quantities in brackets are theoretical predictions for the triplet force for charge $Z=74$ and reduced screening length $\kappa \sigma=0.96$ (see the text).

\begin{tabular}{cccccl}
\hline$R / \sigma$ & Run & \multicolumn{2}{c}{$\begin{array}{c}\text { Triplet force } \\
\text { from theory }\end{array}$} & $\begin{array}{l}\text { Triplet force } \\
\text { from simulation }\end{array}$ & $\begin{array}{l}\text { Statistical } \\
\text { error }\end{array}$ \\
\hline 1.05 & A & -26.1 & & -20.2 & \pm 3 \\
& B & -41.2 & -30.4 & \pm 4 \\
& C & -605 & $(-78.5)$ & -185 & \pm 19 \\
\hline 1.2 & A & -27.1 & & -15.6 & \pm 3 \\
& B & -41.3 & & -25 & \pm 3 \\
& C & -598 & $(-77.8)$ & -139 & \pm 16 \\
\hline 1.5 & A & -22.7 & & -10.3 & \pm 2 \\
& B & -32.7 & & -13.1 & \pm 3 \\
& C & -436 & $(-57.5)$ & -84.6 & \pm 11 \\
\hline 2 & A & -14.9 & & -2.3 & \pm 1 \\
& B & -19.1 & & -8.2 & \pm 2 \\
& C & -221 & $(-29.8)$ & -40.3 & \pm 7 \\
\hline 3 & A & -6.2 & & 2.8 & \pm 1 \\
& B & -6.4 & & -2.2 & \pm 2 \\
& C & -53.7 & $(-7.7)$ & -12.3 & \pm 4 \\
\hline 4 & A & -2.8 & & 6.1 & \pm 2 \\
& B & -2.2 & & 1.9 & \pm 2 \\
& C & -13.7 & $(-2.1)$ & 3.8 & \pm 4 \\
\hline
\end{tabular}

Taking into account the triangle geometry, we get

$$
\begin{gathered}
F^{(\mathrm{DLVO})}=-2 \frac{\boldsymbol{d}}{\boldsymbol{d}} \cdot \nabla_{R}\left(\frac{Z^{2} e^{2}}{\varepsilon} \frac{\exp (-\kappa R)}{R}\right) \\
F_{\text {pert }}^{(2)}=2 \frac{\boldsymbol{d}}{\boldsymbol{d}} \cdot \nabla_{\boldsymbol{R}_{1}}\left[\frac { k _ { B } T } { 6 } \int _ { \Omega } \mathrm { d } ^ { 3 } r \left\{\frac{1}{\rho_{+}^{2}}\left[\frac{2 \rho_{+} \rho_{-}}{\rho_{+}+\rho_{-}}+\frac{\kappa_{+}^{2}}{\kappa^{2}} \sum_{j=1}^{2} \varphi\left(\left|\boldsymbol{r}-\boldsymbol{R}_{j}\right|\right)-\rho_{+}\right]^{3}\right.\right. \\
\left.\left.+\frac{1}{\rho_{-}^{2}}\left[\frac{2 \rho_{+} \rho_{-}}{\rho_{+}+\rho_{-}}-\frac{\kappa_{-}^{2}}{\kappa^{2}} \sum_{j=1}^{2} \varphi\left(\left|\boldsymbol{r}-\boldsymbol{R}_{j}\right|\right)-\rho_{-}\right]^{3}\right\}\right]
\end{gathered}
$$

and

$$
F_{\text {pert }}^{(3)}=\frac{\boldsymbol{d}}{d} \cdot \nabla_{\boldsymbol{R}_{1}}\left[\frac{k_{B} T}{\left(\rho_{+}+\rho_{-}\right)^{2}} \int_{\Omega} \mathrm{d}^{3} r \varphi\left(\left|\boldsymbol{r}-\boldsymbol{R}_{1}\right|\right) \varphi\left(\left|\boldsymbol{r}-\boldsymbol{R}_{2}\right|\right) \varphi\left(\left|\boldsymbol{r}-\boldsymbol{R}_{3}\right|\right)\right]
$$

with the orbital given by (30). It is obvious that the forces (32), (33) will only depend on the triangle length $R$. Hence the perturbation theory also alters the pairwise part with respect to DLVO theory.

\section{Results}

The simulation parameters were chosen to be typical for charged colloidal suspensions: $T=293 \mathrm{~K}, \rho_{m}=3.3 \times 10^{12} \mathrm{~cm}^{-3}, \sigma \equiv 2 R_{m}=1.108 \times 10^{-5} \mathrm{~cm}, q_{i}= \pm 1$. The diameter of the macro-ion, $\sigma \equiv 2 R_{m}$, and the parameter $e^{2} / \sigma^{2}$ were used as dimensionless 


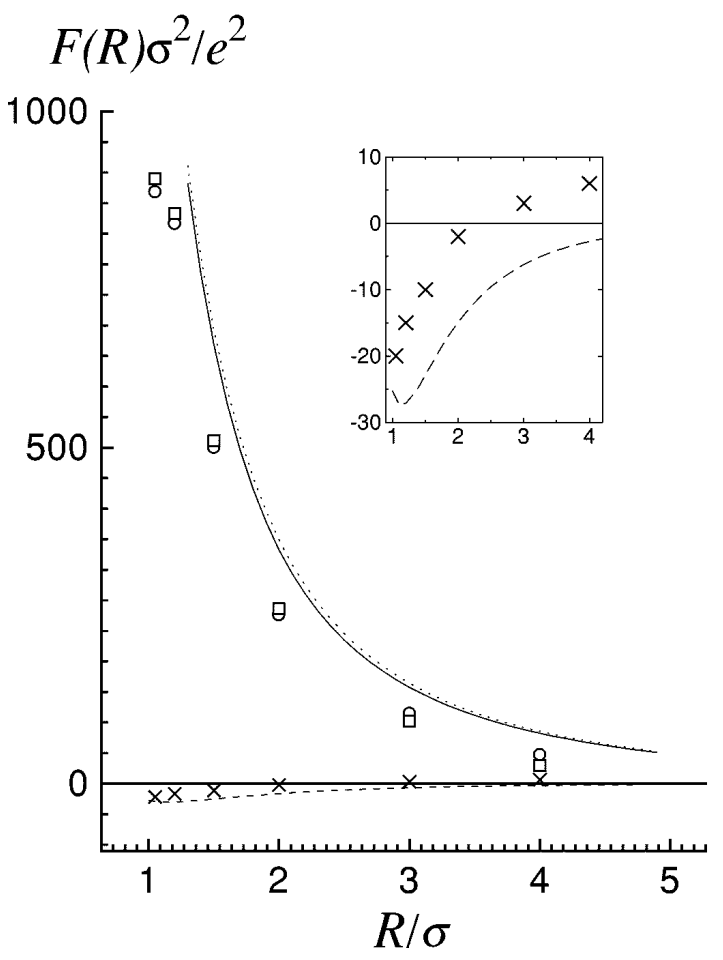

Figure 2. The effective force $F(R)$ (in units of $e^{2} / \sigma^{2}$ ) acting on the first macro-ion versus reduced distance $R / \sigma$ for run A. Open circles: the computer simulation for the total force. Open squares: the computer simulation for the pairwise part. Crosses: the difference between those two quantities. Dotted line: the pairwise DLVO prediction. Dashed line: the effective threeparticle interaction from perturbation theory. Solid line: the total effective force as obtained from theory. The inset shows the triplet forces on a larger scale.

measures for the distance and the effective force. In these units, the length of the cubic cell is $L / \sigma=8.74$. We choose $\sigma_{+}, \sigma_{-}=10^{-3} \sigma$.

The following sets of the parameters have been examined:

$$
\begin{array}{ll}
\text { Run A: } & Z=280, \varepsilon=81, \rho_{s}=0 \\
\text { Run B: } & Z=280, \varepsilon=81, \rho_{s}=\rho_{c} / 3 \\
\text { Run C: } & Z=200, \varepsilon=15, \rho_{s}=0 .
\end{array}
$$

Run A was done with $N_{c}=840$ counter-ions; run C was carried out for $N_{c}=600$ counter-ions. Run B with added salt ions took most simulation time, and involved a total number of $N_{c}+N_{s}=1680$ microscopic ions.

The equilibrium state of the system was checked during the simulation time for every run. This was done by monitoring the temperature, average velocity and the distribution function of velocities and total potential energy of the system throughout. On average, it took about $2000 \mathrm{MD}$ steps to get to equilibrium. Then during 20000-50000 time steps we gathered statistics to perform the canonical averages in equation (8). The time step $\Delta t$ was typically chosen to be $5 \times 10^{-3} \sqrt{m \sigma^{3} / e^{2}}$, such that a counter-ion with average velocity traverses a path of about $\sigma / 100$. By comparing averages over statistically independent runs, we estimated the statistical error of the force; see table 1. It was found to be significantly smaller than the symbol size in the figures. 


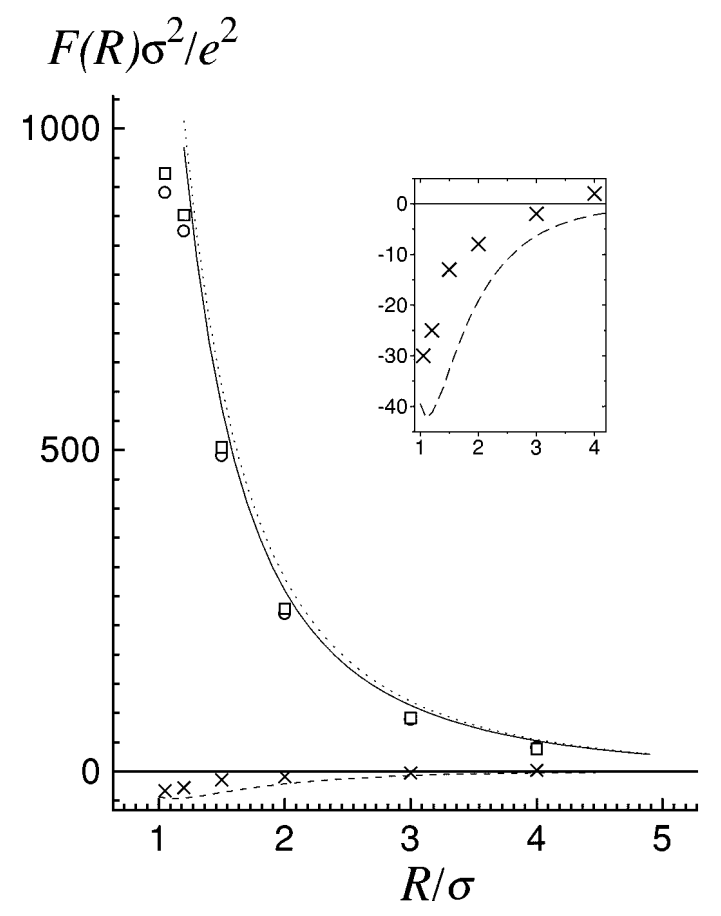

Figure 3. As figure 2, but now for the added-salt case (run B).

Figure 2 shows the computer simulation results for $F(R)$ as well as those obtained from density functional perturbation theory for run A. The open circles show the full simulation data involving pairwise and triplet contributions. If one determines the interaction between two particles alone by a reference calculation as in references $[14,16]$, then one gets the pairwise force $F^{(2)}(R)$. Hence the total contribution of these pairwise forces in the equilateral triangle configuration is $\sqrt{3} F^{(2)}(R)$. This quantity is included also in figure 2 (open squares). The difference between $F(R)$ and $\sqrt{3} F^{(2)}(R)$ is the pure triplet contribution which is marked by crosses in figure 2 . It becomes clear that this contribution is negative for small separations, and hence the additional triplet interaction is attractive. For larger separation, one has to be careful with wall-induced effects which make a direct interpretation of the sign of the force more difficult.

We have also included the corresponding density functional perturbation results in figure 2. The pure triplet part $F_{\text {pert }}^{(3)}(r)$ is given as a broken line, while the perturbation results for the total forces are given as a solid line. To complete the story, we have shown also the corresponding pairwise contribution $F_{\text {pair }}=F^{(\mathrm{DLVO})}+F_{\text {pert }}^{(2)}$ (dotted line). Comparing theory and computer simulation data, it becomes clear that the theory describes the attractive nature of the triplet forces for nearly touching macro-ions, although it overestimates the strength of this attraction; see the inset of figure 2 and table 1 .

The same conclusions apply to a situation with added salt (run B) which is shown in figure 3; see again table 1. Again a reasonable agreement for the total effective force was found here. It should be noted, however, that the situations of run A and B both correspond to rather weak coupling, and hence that the DLVO pair theory already works reasonably well. It is anticipated, of course, that the perturbation approach then holds, since the difference between the DLVO theory and the computer simulation data is actually 


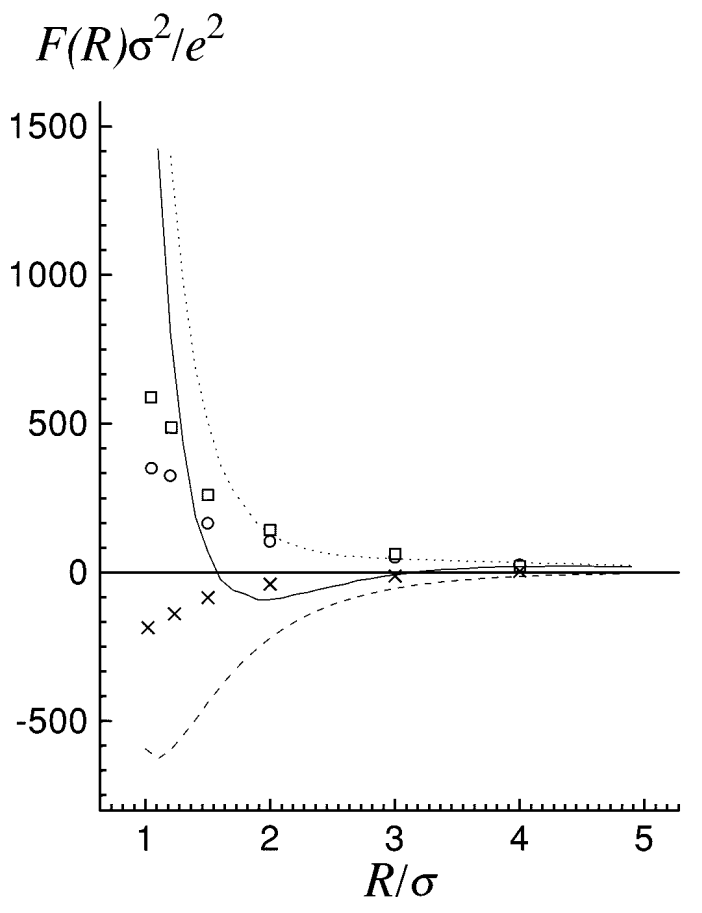

Figure 4. As figure 2, but now for the strong-coupling case (run C).

small. Quite different behaviour is seen for run C in figure 4. Here the dielectric constant $\varepsilon$ was reduced from 81 to 15 . This leads to large deviations between DLVO theory and the computer simulation data. The strong coupling is demonstrated also by a typical counter-ion snapshot configuration shown in figure 5 for two different separations $R$. Of course then also our perturbation approach has to break down and the total force becomes even negative, in disagreement with simulation; see again figure 4 . This deficiency of the theory can be cured by fitting the pairwise computer simulation data by an expression involving the total pair terms, $F^{(\mathrm{DLVO})}+F_{\text {pert }}^{(2)}$, with two free fitting parameters $Z^{*}$ and $\kappa^{*}$ in the orbital function $\varphi(r)$, equation (30). The best fit was obtained for a strongly reduced effective charge $Z^{*}=74$ and $\kappa^{*}=0.96$ and is shown as a dotted line in figure 6 . The renormalization of the charge is consistent with earlier findings [11, 13]. Using these 'renormalized' parameters in our perturbation theory (27), the triplet forces are shown as a broken line in figure 6; see also table 1 . Now the agreement is reasonable both for the pure triplet part and for the total force except close to touching. This indicates that the perturbation approach is still useful for inter-macro-ion distances that are not too small, once one knows the effective parameters for the pairwise interaction. The Poisson-Boltzmann cell model [11], for instance, which is designed for strong coupling, provides the basis for such an approach for obtaining $Z^{*}$ and $\kappa^{*}$ theoretically.

Hence to get an analytical expression for the triplet force in strongly coupled systems one has to proceed as follows.

(i) Get the parameters $Z^{*}, \kappa^{*}$ from a theory, e.g. the Poisson-Boltzmann cell model.

(ii) Use the expression

$$
\frac{k_{B} T}{\left(\rho_{+}+\rho_{-}\right)^{2}} \int_{\Omega} \mathrm{d}^{3} \boldsymbol{r} \varphi\left(\left|\boldsymbol{r}-\boldsymbol{R}_{1}\right|\right) \varphi\left(\left|\boldsymbol{r}-\boldsymbol{R}_{2}\right|\right) \varphi\left(\left|\boldsymbol{r}-\boldsymbol{R}_{3}\right|\right)
$$




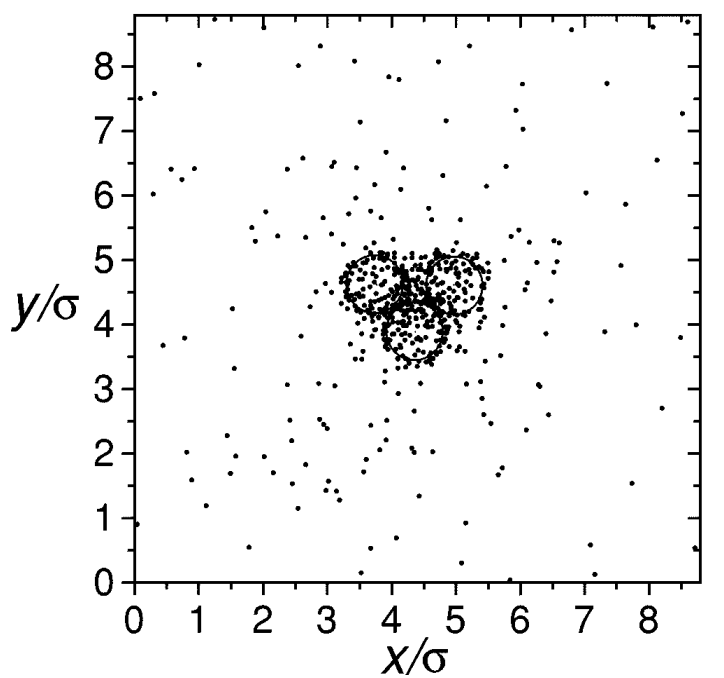

(a)

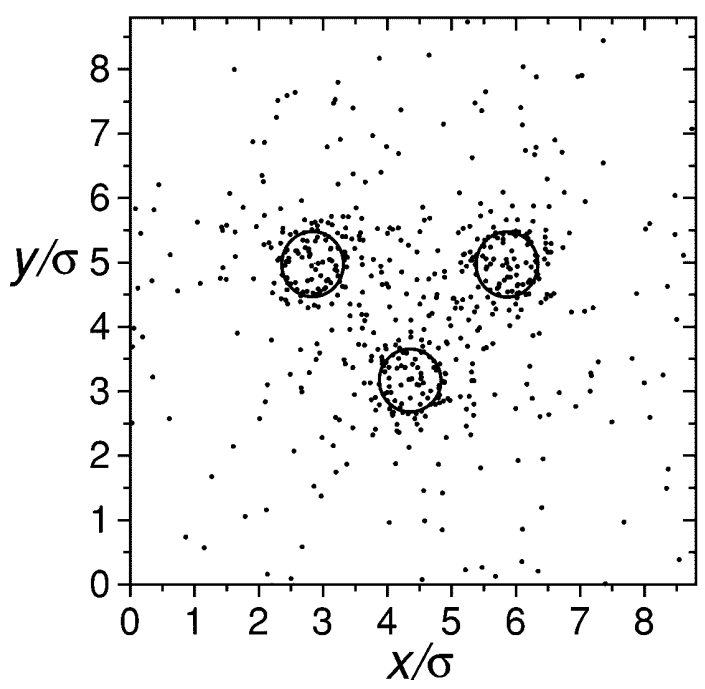

(b)

Figure 5. A snapshot picture (projected onto the $x y$-plane) from run $\mathrm{C}$. The big open circles correspond to the cores of the macro-ions. The dots are the counter-ions. (a) For $R=1.2 \sigma$. (b) For $R=3 \sigma$.

for the triplet potential where

$$
\varphi(r)= \begin{cases}\frac{Z^{*} e^{2}}{\varepsilon r} \exp \left(-\kappa^{*} r\right) & \text { for } r \geqslant R_{m} \\ 0 & \text { for } r \geqslant R_{m} .\end{cases}
$$

We finally remark that the triplet forces become relevant for strong Coulomb coupling and nearly touching configurations. For run $\mathrm{C}$ (see again figures 4 and 6) they have the same order of magnitude as the pairwise forces. 


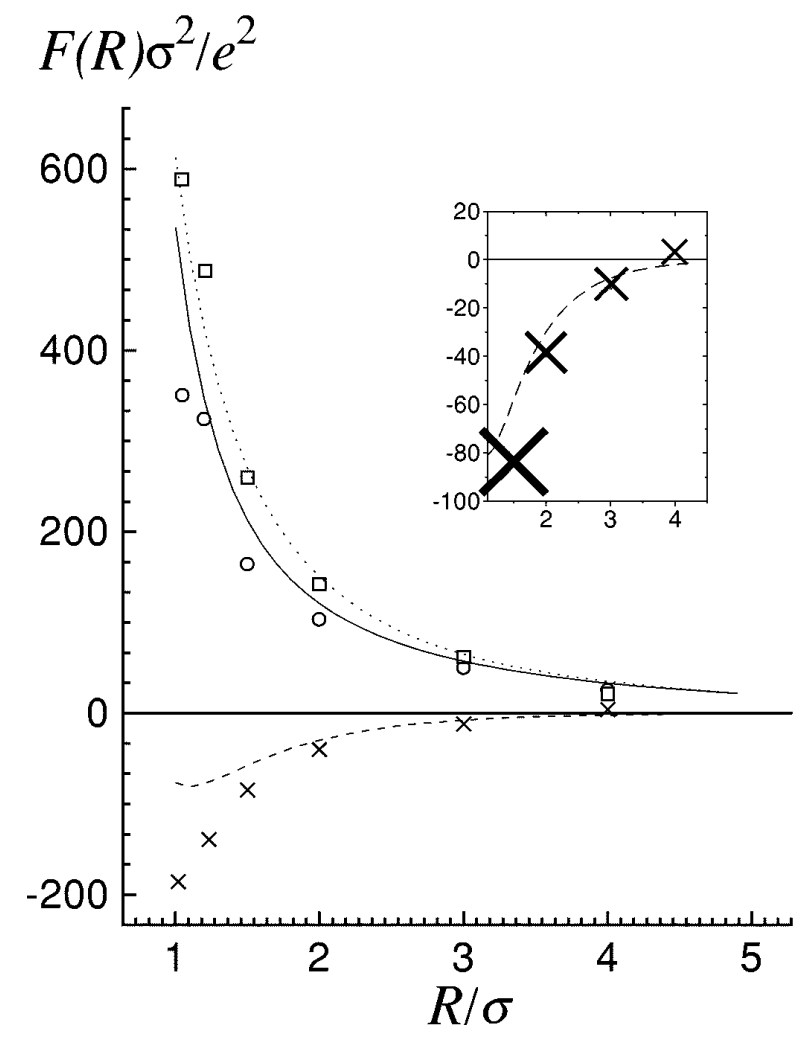

Figure 6. As figure 4, but with renormalized theoretical input. The dotted line is the best fit for the pairwise part. The broken line is the triplet contribution obtained with these fitting parameters and the solid line is the resulting total force as obtained from renormalized theory. The inset shows the triplet forces on a larger scale.

\section{Discussion and forward look}

To summarize: we have calculated, by 'exact' computer simulation applied to the primitive model, the effective triplet forces between macro-ions in a triplet configuration in the form of an equilateral triangle. As a result, the triplet contribution gives rise to an additional attraction as compared to the pure pairwise repulsive terms. The correction is particularly significant for small distances in the macro-ion triplet. We also derived and proposed an analytical expression for the triplet interaction potential, which is a cube of a linear superposition of Yukawa orbitals for the ion density. This expression was checked against our computer simulation data and fair agreement was found for small couplings (run A and B). For strong coupling this expression is only valid if the parameters are rescaled and 'renormalized' such that the pairwise parts are reproduced well by a Yukawa form of the effective potential.

For our parameter combinations the net force was always repulsive, although the pure triplet contribution was attractive. It would be interesting to study situations of extreme coupling where the presence of a third particle in fact induces an attraction among the triplet triangle particles. Such a situation would immediately result in coagulation of particle triplets. Work along these lines is in progress. 


\section{Acknowledgments}

This work was supported by the DFG within the SFB 237 (Unordnung und grosse Fluktuationen). We thank Professor S A Trigger and H Graf for fruitful conversations and discussions of the results of this article.

\section{References}

[1] See, e.g.,

Hansen J-P and McDonald I R 1986 Theory of Simple Liquids 2nd edn (London: Academic)

[2] See, e.g.,

Allen M P and Tildesley D J 1989 Computer Simulation of Liquids (Oxford: Clarendon)

[3] Löwen H 1994 Phys. Rep. 237249

[4] Derjaguin B V and Landau L D 1941 Acta Physicochim. USSR 14633

[5] Verwey E J W and Overbeek J T G 1948 Theory of the Stability of Lyophobic Colloids (Amsterdam: Elsevier)

[6] Rowlinson J S 1984 Mol. Phys. 52567

[7] Grimson M J and Silbert M 1991 Mol. Phys. 74397

[8] Löwen H, Hansen J-P and Madden P A 1993 J. Chem. Phys. 983275

[9] Axilrod B M and Teller E 1943 J. Chem. Phys. 11229

[10] Tau M, Reatto L, Magli R, Egelstaff P A and Barocchi F 1989 J. Phys.: Condens. Matter 17131

[11] Alexander S, Chaikin P M, Grant P, Morales G J, Pincus P and Hone D 1984 J. Chem. Phys. 805776

[12] Löwen H, Madden P A and Hansen J-P 1992 Phys. Rev. Lett. 681081

[13] Löwen H and Kramposthuber G 1993 Europhys. Lett. 23673

[14] D'Amico I and Löwen H 1997 Physica A 23725 Löwen H and D'Amico I 1997 J. Phys.: Condens. Matter 98879

[15] Allahyarov E A and Trigger S A 1998 Physica at press

[16] Allahyarov E A, Löwen H and Trigger S A 1988 Phys. Rev. E at press

[17] See, e.g.,

Barrat J L, Hansen J-P and Pastore G 1987 Phys. Rev. Lett. 582075

[18] Linse P 1991 J. Chem. Phys. 948227 